\title{
Previsão pré-operatória de fraturas supracondilares de úmero Gartland IV: É possível?*
}

\section{Pre-operative Prediction of Gartland IV Supracondylar Fractures of Humerus: Is it Possible?}

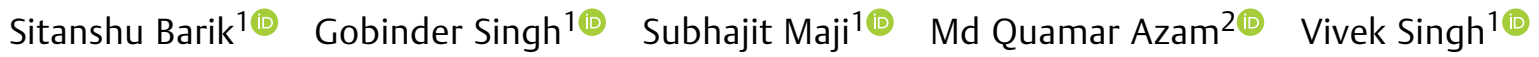 \\ ${ }^{1}$ Departamento de Ortopedia, All India Institute of Medical Sciences, \\ Rishikesh, Índia \\ 2 Departamento de Cirurgia de Trauma, All India Institute of Medical \\ Sciences, Rishikesh, Índia \\ Endereço para correspondência Gobinder Singh, M.B.B.S., Residente \\ Júnior, Ortopedia, Sala 84219, Edifício 84, AlIMS Rishikesh, Dehradun, \\ Uttarakhand, Índia (e-mail: gobindergs@gmail.com). \\ Rev Bras Ortop 2021;56(2):230-234.
}

\section{Resumo \\ Palavras-chave \\ - fraturas do úmero \\ - criança \\ - articulação do cotovelo \\ - fixação de fratura}

Objetivos $\mathrm{O}$ presente estudo tem como objetivo identificar características préoperatórias do paciente e da lesão, bem como da imagem que apontaria para uma fratura tipo IV. O presente estudo ajudará a equipe operacional a prever com mais precisão o padrão tipo IV pré-operatório, levando a um melhor aconselhamento dos cuidadores e planejamento da cirurgia, bem como a uma melhor preparação em relação à redução aberta, se tal situação surgir.

Métodos Um estudo retrospectivo foi realizado, incluindo pacientes que atendiam os seguintes critérios: 1 ) idade $<16$ anos; 2) fraturas supracondilares Gartland tipos III e IV; e 3) com registros completos. Foram coletados dados demográficos como idade, gênero, lateralidade, modo de lesão, duração hospitalar de lesão, histórico de tentativas anteriores de redução fechada, fratura aberta/fechada, estado neurovascular distal e dados radiográficos como angulação, translação, aposição óssea e cominação de fratura.

Resultados A duração hospitalar de lesões e as tentativas anteriores de redução fechada foram os fatores com diferença estatisticamente significativa entre as fraturas tipo III e IV $(p<0,05)$. O diagnóstico de fraturas supracondilares tipo IV foi significativamente mais provável na presença de angulação em valgo de fragmento distal $\geq 17^{\circ}$ (odds ratio $[O R]=20,22$; intervalo de confiança $[I C] 95 \%=3,45-118,65$ ). A angulação de flexão $\geq 10^{\circ}(\mathrm{OR}=5,32 ; \quad \mathrm{IC} 95 \%=0,24-119,88)$ do fragmento distal previram Gartland tipo IV com sensibilidade de $41 \%$ e especificidade de $100 \%$.

Conclusão A avaliação pré-operatória de suspeitas de fraturas de Gartland IV pode ajudar o cirurgião operacional a prever tais lesões. Fatores não radiográficos, como o

Trabalho desenvolvido no All India Institute of Medical Sciences, Rishikesh, Índia.

recebido

28 de Abril de 2020

aceito

17 de Setembro de 2020
DOI https://doi.org/

$10.1055 / \mathrm{s}-0040-1722578$ ISSN 0102-3616.
(C) 2021. Sociedade Brasileira de Ortopedia e Traumatologia. All rights reserved.

This is an open access article published by Thieme under the terms of the Creative Commons Attribution-NonDerivative-NonCommercial-License, permitting copying and reproduction so long as the original work is given appropriate credit. Contents may not be used for commercial purposes, or adapted, remixed, transformed or built upon. (https://creativecommons.org/ licenses/by-nc-nd/4.0/)

Thieme Revinter Publicações Ltda., Rua do Matoso 170, Rio de Janeiro, RJ, CEP 20270-135, Brazil 


\section{Abstract}

\section{Keywords}

- humeral fractures

- child

- elbow joint

- fracture fixation aumento da duração da lesão hospitalar, tentativas de redução previamente fechada e parâmetros radiográficos como valgo e angulação de flexão foram mais propensos a estarem associados a fraturas tipo IV. Nível de evidência III.

Objectives The present study aims to identify preoperative characteristics of the patient, of the injury, as well as of imaging, which would point towards a type IV fracture. The present study shall help the operating team to predict more accurately the type IV pattern preoperatively, leading to improved counselling of the caregivers, planning of surgery, as well as preparedness regarding open reduction, if such situation arises.

Methods A retrospective study was conducted, including patients that met the following criteria: 1 ) age $<16$ years old; 2 ) Gartland type-III and type-IV supracondylar fractures; and 3) with complete records. Demographic data like age, gender, laterality, mode of injury, hospital duration of the injury, history of previous attempts of closed reduction, open/closed fracture, distal neurovascular status, and radiographic data like angulation, translation, osseous apposition and fracture comminution were collected. Results Hospital duration of the injury and previous attempts of closed reduction were the factors that had a statistically significant difference among types III and IV fractures $(p<0.05)$. A diagnosis of type IV supracondylar fractures was significantly more likely in the presence of valgus angulation of the distal fragment $\geq 17^{\circ}$ (odds ratio $[O R]=20.22 ; 95 \%$ confidence interval $[\mathrm{Cl}]=3.45-118.65$ ). Flexion angulation $\geq 10^{\circ}$ $(\mathrm{OR}=5.32 ; 95 \% \mathrm{Cl}=0.24-119.88)$ of the distal fragment predicted Gartland type IV with a sensitivity of $41 \%$ and a specificity of $100 \%$.

Conclusion The preoperative evaluation of suspected Gartland IV fractures can help the operating surgeon in predicting such injuries. Nonradiographic factors like increased hospital duration of the injury, attempts at previously closed reduction, and radiographic parameters like valgus and flexion angulation were more likely to be associated with type IV fractures. Level of evidence III.

\section{Introdução}

Fraturas supracondilares são algumas das fraturas comuns ao redor do cotovelo pediátrico. Elas compreendem cerca de dois terços de todo o trauma pediátrico em torno do cotovelo que requer internação, com uma incidência estimada em 177,3 por 100.000 crianças. $^{1}$ Tais fraturas são comumente observadas em meninos na faixa etária de 5 a 8 anos, com o braço não dominante sendo comumente envolvido. ${ }^{2} 0$ sistema mais utilizado para classificar tais fraturas é a classificação Gartland, que tem uma boa concordância interobservador e intraobservador. ${ }^{3}$ Leitch introduziu um tipo IV à classificação Gartland, no qual o periósteo anterior e o posterior são completamente arrancados, levando a instabilidade tanto na flexão quanto na extensão. ${ }^{4}$ Esses tipos de fraturas são considerados diagnosticáveis apenas intraoperatoriamente., ${ }^{1,5}$

Embora a redução fechada e fixação percutânea (RFFP) seja a modalidade comum de tratamento para as fraturas de Gartland tipo III e IV, a redução dos casos do tipo IV pode ser difícil devido à instabilidade multidirecional inerente. As fraturas tipo IV podem precisar mais comumente de uma redução aberta e fixação percutânea (RAFP) em comparação com as fraturas tipo III. ${ }^{5}$ Há pouca litera- tura sobre o diagnóstico e o tratamento ideal das fraturas tipo IV. O presente estudo tem como objetivo identificar características pré-operatórias do paciente, da lesão, bem como da imagem que apontariam para uma fratura tipo IV. O presente estudo ajudará a equipe operacional a prever com mais precisão o padrão tipo IV no pré-operatório, levando a um melhor aconselhamento dos cuidadores, melhor planejamento da cirurgia, bem como a uma melhor preparação em relação à redução aberta, se tal situação surgir.

\section{Material e Métodos}

Após a aprovação pelo Comitê de Ética Institucional para estudo observacional retrospectivo, foi adquirida a base de dados de pacientes para os casos que se apresentaram ao departamento de ortopedia para o tratamento de fraturas supracondilares de janeiro de 2016 a dezembro de 2019. Os pacientes incluídos no presente estudo atendiam aos seguintes critérios: 1 ) idade $<16$ anos; 2) fraturas supracondilares Gartland tipos III e IV; e 3) com registros completos. Foram excluídos do estudo pacientes com 1) outra lesão concomitante no mesmo membro; 2) 

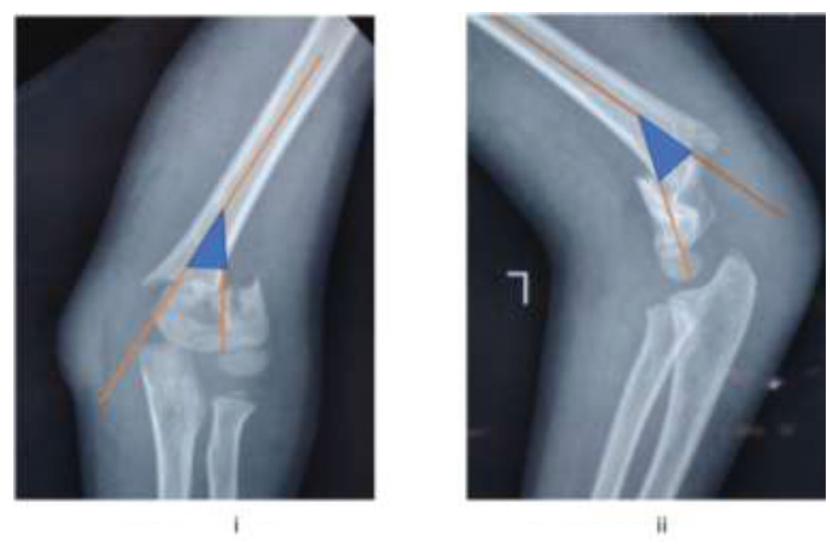

Fig. 1 (i, ii). Representação de angulação no plano coronal (i) e sagital (ii) por linhas ao longo da diáfise média do fragmento proximal e linha perpendicular à articulação do cotovelo no fragmento distal.

histórico de trauma anterior no cotovelo; 3) suspeita de displasia esquelética; e 4) registros incompletos.

Idade, gênero, lateralidade, modo de lesão, duração hospitalar da lesão, histórico de tentativas anteriores de redução fechada, fratura aberta/fechada e estado neurovascular distal foram determinados por meio da revisão dos prontuários.

Radiografias pré-operatórias foram avaliadas para avaliar os seguintes parâmetros: 1) angulação do fragmento distal no plano sagital; 2) angulação do fragmento distal no plano coronal ( - Fig. 1); 3) translação do fragmento distal no plano coronal; 4) translação do fragmento distal no plano sagital (-Fig. 2); 5) aposição óssea entre os fragmentos proximais e distais no plano anteroposterior; 6) aposição óssea entre os fragmentos proximais e distais em radiografias laterais (-Fig. 2); e 7) cominação de fratura. Notas operacionais foram examinadas para determinar o tipo Gartland de fraturas supracondilares.

Os dados foram analisados por meio do teste qui-quadrado ou dos testes exatos de Fisher para variáveis categóricas e do teste $t$ amostral para variáveis contínuas. As razões de chances (ORs, na sigla em inglês) foram estimadas e relatadas com intervalos de confiança (ICs) associados de $95 \%$. As curvas ROC foram exploradas para comparação do desempenho diagnós-

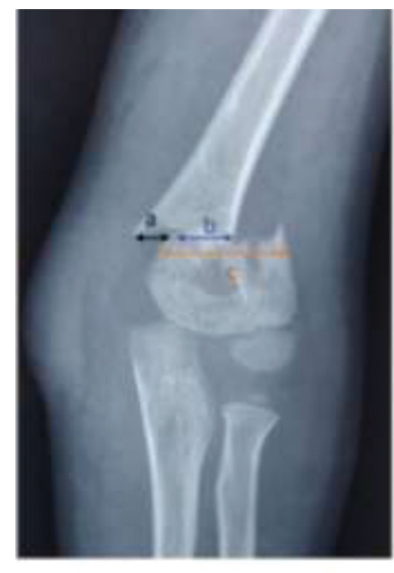

1

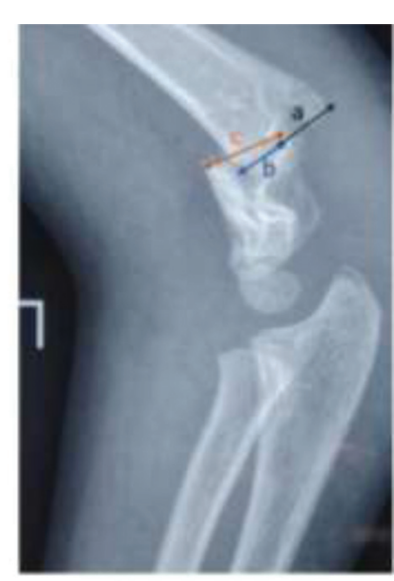

ii
Fig. 2 (i, ii). Representação da translação e aposição óssea em plano coronal (i) e sagital (ii). Translação =a / c. Aposição óssea =b / c. tico de vários parâmetros radiográficos na previsão das fraturas tipo IV de Gartland. A análise dos dados foi realizada utilizando-se o software IBM SPSS Statistics for Windows, versão 24.0 (IBM Corp., Armonk, NY, EUA).

\section{Resultados}

Foram incluídos no presente estudo 33 pacientes (25 do sexo masculino, 8 do sexo feminino) que atenderam aos critérios do estudo, com idade média de $8,7 \pm 3,1$ anos ( Tabela 1). Os modos de lesão mais comuns ocorreram quando os pacientes praticavam esportes ou caíram de lugares altos. A fratura foi encerrada em $96,9 \%(n=32)$ dos casos. Entre os pacientes incluídos no estudo, 16 foram de Gartland tipo III, e 17 de Gartland tipo IV.

A duração hospitalar de lesões e as tentativas anteriores de redução de fechamento foram os fatores com diferença estatisticamente significante entre as fraturas tipo III e IV ( - Tabela 1). A duração de estadia no hospital da lesão média na fratura supracondilar tipo III foi de 31,25 $\pm 43,09$ horas, enquanto no tipo IV ela foi de $52,59 \pm 45,47$ horas. Um histórico de tentativas anteriores de redução de fechamento

Tabela 1 Associação entre tipo Gartland e parâmetros

\begin{tabular}{|c|c|c|c|}
\hline \multirow[t]{2}{*}{ Parâmetros } & \multicolumn{2}{|l|}{ Tipo Gartland } & \multirow[t]{2}{*}{ valor $=p$} \\
\hline & $\begin{array}{l}\text { Tipo } 3 \\
(n=16)\end{array}$ & $\begin{array}{l}\text { Tipo } 4 \\
(n=17)\end{array}$ & \\
\hline Idade (Anos) & $8,62 \pm 2,60$ & $8,88 \pm 3,69$ & $0,818^{\mathrm{a}}$ \\
\hline \multicolumn{3}{|l|}{ Gênero } & \multirow[t]{3}{*}{$1,000^{b}$} \\
\hline Masculino & $12(75,0 \%)$ & $13(76,5 \%)$ & \\
\hline Fêminino & $4(25,0 \%)$ & $4(23,5 \%)$ & \\
\hline \multicolumn{3}{|l|}{ Modo de lesão } & \multirow[t]{4}{*}{$0,071^{\mathrm{b}}$} \\
\hline Queda de altura & $9(56,2 \%)$ & $6(35,3 \%)$ & \\
\hline $\begin{array}{l}\text { Lesão enquanto } \\
\text { pratica esportes }\end{array}$ & $5(31,2 \%)$ & $11(64,7 \%)$ & \\
\hline $\begin{array}{l}\text { Acidente } \\
\text { de trânsito }\end{array}$ & $2(12,5 \%)$ & $0(0,0 \%)$ & \\
\hline $\begin{array}{l}\text { Intervalo } \\
\text { lesão-hospital } \\
\text { (horas) }^{* * *}\end{array}$ & $31,25 \pm 43,09$ & $52,59 \pm 45,47$ & $0,029^{c}$ \\
\hline \multicolumn{3}{|l|}{ Lateralidade } & \multirow[t]{3}{*}{$0,392^{d}$} \\
\hline Direita & $9(56,2 \%)$ & $12(70,6 \%)$ & \\
\hline Esquerda & $7(43,8 \%)$ & $5(29,4 \%)$ & \\
\hline $\begin{array}{l}\text { Tentativas } \\
\text { anteriores } \\
\text { de redução } \\
\text { fechada } \\
\text { (presente) }\end{array}$ & $4(25,0 \%)$ & $11(64,7 \%)$ & $0,022^{d}$ \\
\hline \multicolumn{3}{|l|}{ Tipo de fratura } & \multirow[t]{3}{*}{$0,485^{\mathrm{b}}$} \\
\hline Fechada & $15(93,8 \%)$ & $17(100,0 \%)$ & \\
\hline Aberta & $1(6,2 \%)$ & $0(0,0 \%)$ & \\
\hline \multicolumn{3}{|l|}{ DNVS } & \multirow[t]{3}{*}{$0,335^{\mathrm{b}}$} \\
\hline Intacto & $13(81,2 \%)$ & $16(94,1 \%)$ & \\
\hline Envolvido & $3(18,8 \%)$ & $1(5,9 \%)$ & \\
\hline
\end{tabular}

${ }^{* * *}$ Significativo em $p<0,05,{ }^{\text {a }}$ : teste-t, ${ }^{\text {b}}$ : Teste exato de Fisher, ${ }^{\mathrm{c}}$ : Teste de Wilcoxon, d: Teste qui-quadrado. DNVS, Distal neurovascular status. 
Tabela 2 Comparação do desempenho diagnóstico de vários parâmetros radiográficos para predizer Gartland Tipo 4 versus Gartland Tipo 3

\begin{tabular}{|c|c|c|c|c|c|c|c|}
\hline Preditor & Razão ímpar (IC 95\%) & AUROC (IC95\%) & valor-p & Sn & Sp & PPV & NPV \\
\hline $\begin{array}{l}\text { Angulação (plano sagital) } \\
\text { (graus de extensão) }\end{array}$ & $5,32(0,24-119,88)$ & $0,557(0,343-0,771)$ & 0,587 & $41 \%$ & $100 \%$ & $100 \%$ & $62 \%$ \\
\hline $\begin{array}{l}\text { Angulação } \\
\text { (plano coronal) (valgo) }\end{array}$ & $20,22(3,45-118,65)$ & $0,868(0,741-0,994)$ & $<0,001$ & $88 \%$ & $81 \%$ & $83 \%$ & $87 \%$ \\
\hline Translação (plano coronal) (\%) & $0,94(0,02-50,32)$ & $0,557(0,344-0,77)$ & 0,588 & $71 \%$ & $69 \%$ & $71 \%$ & $69 \%$ \\
\hline Translação (plano sagital) (\%) & $3,14(0,75-13,16)$ & $0,515(0,316-0,714)$ & 0,897 & $41 \%$ & $69 \%$ & $58 \%$ & $52 \%$ \\
\hline Aposição óssea (AP) (\%) & $3,14(0,75-13,16)$ & $0,557(0,344-0,77)$ & 0,588 & $71 \%$ & $69 \%$ & $71 \%$ & $69 \%$ \\
\hline Aposição óssea (Lateral) (\%) & $0,94(0,02-50,32)$ & $0,515(0,316-0,714)$ & 0,897 & $41 \%$ & $69 \%$ & $58 \%$ & $52 \%$ \\
\hline
\end{tabular}

Abreviaturas: AUROC, área sob curva ROC; IC, intervalo de confiança; DA, precisão diagnóstica; NPV, valor preditivo negativo; PPV, Valor preditivo positivo; Sn, sensibilidade; Sp, especificidade.

esteve presente em 11 pacientes (64,7\%) de fraturas supracondilares tipo IV, enquanto estava presente em 4 (25\%) pacientes de fraturas tipo III. Outros fatores demográficos como idade, gênero, lateralidade, fratura aberta/fechada e estado neurovascular distal não foram estatisticamente significativos na diferenciação de fraturas tipos III e IV.

Foram analisadas 33 radiografias pré-operatórias, e o diagnóstico de fraturas supracondilares tipo IV foi significativamente mais provável na presença de angulação de valgo de fragmento distal $\geq 17^{\circ}(\mathrm{OR}=20,22$; IC95\% $=3,45$ $118,65)$, com sensibilidade de $88 \%$ e especificidade de $81 \%$ (-Tabela 2, - Fig. 3). A angulação de flexão $\geq 10^{\circ}(\mathrm{OR}=5,32$; IC95\% $=0,24-119,88)$ do fragmento distal prevê Gartland tipo IV com sensibilidade de $41 \%$ e especificidade de $100 \%$. Não houve associação significativa com a cominação da fratura, com a translação do fragmento distal sobre o fragmento proximal em ambos os planos, tampouco com a aposição óssea entre fragmentos de fratura em ambos os planos na previsão de fraturas supracondilares tipo IV em radiografias pré-operatórias (-Tabela $\mathbf{2}$, - Fig. $\mathbf{3}$ ).

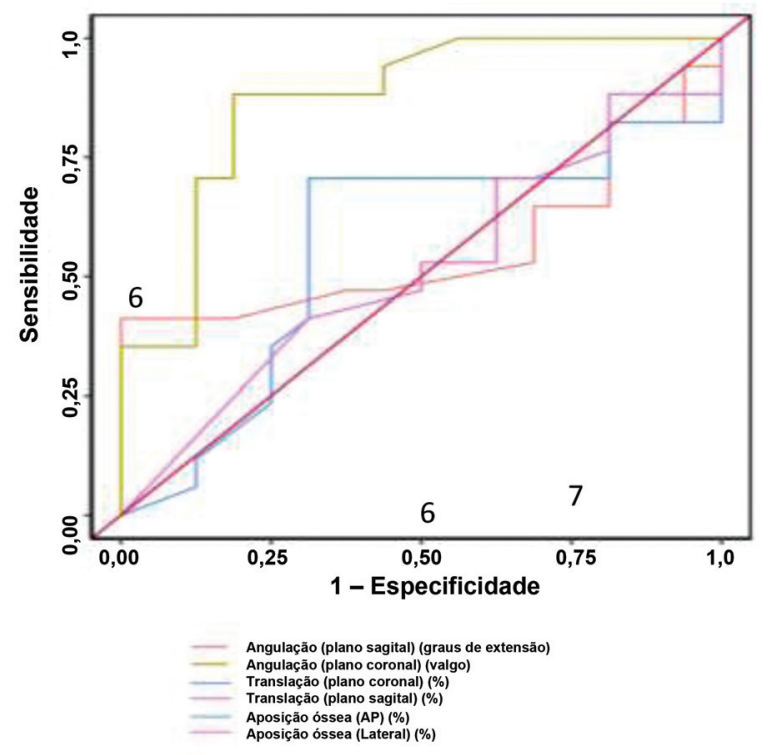

Fig. 3 Análise de curva ROC de vários parâmetros para prever Gartland tipo 4 versus Gartland tipo 3.

\section{Discussão}

O tipo multidirecionalmente instável de fratura supracondilar foi descrito pela primeira vez por Leitch em $2006 .{ }^{4}$ Tais fraturas não têm nenhuma dobradiça periosteal, e o fragmento distal é instável tanto na flexão quanto na extensão. Esse tipo de fratura foi renomeado como Gartland tipo IV pelos autores. A incidência de tais fraturas varia de 7 a $10 \%$ de todas as fraturas supracondilares operadas. ${ }^{4}$ Do pool de fraturas instáveis operadas incluídas no presente estudo, $\sim 50 \%(n=17)$ eram do tipo IV. $O$ diagnóstico deste tipo de fratura é possível intraoperatoriamente, e as capacidades intraobservador e interobservador ainda são indeterminadas. Essa instabilidade também pode ficar exagerada durante as tentativas de redução, o que confunde ainda mais a taxa real de ocorrência. ${ }^{4}$ Por fim, o diagnóstico da fratura tipo IV é extremamente subjetivo e depende do cirurgião.

O presente estudo observou uma diferença significativa na duração hospitalar da lesão e nas tentativas anteriores de redução fechada na previsão da fratura tipo IV em vez da fratura tipo III. A duração de estadia no hospital da lesão média na fratura supracondilar tipo III foi de 31,25 $\pm 43,09$ horas, enquanto, no tipo IV, ela foi de $52,59 \pm 45,47$ horas. Um histórico de tentativas anteriores de redução de fechamento esteve presente em 11 pacientes (64,7\%) de fraturas supracondilares tipo IV, enquanto esteve presente em 4 (25\%) pacientes de fraturas tipo III. Lesões neurovasculares não são incomuns em fraturas tipo IV e variam em torno de 0 a $36 \%{ }^{4,6}$ O presente estudo apresentou um único paciente de fratura tipo IV com lesão neurovascular. Outros fatores demográficos como idade, gênero, lateralidade, fratura aberta/fechada e estado neurovascular distal não foram estatisticamente significativos na diferenciação de fraturas tipo III e IV.

O presente estudo tentou identificar os parâmetros radiográficos pré-operatórios que seriam mais propensos a estarem associados a fraturas tipo IV. $\mathrm{O}$ fator mais estatisticamente significativo foi a angulação em valgo do fragmento distal (sensibilidade de $88 \%$, especificidade de $81 \%$ ). A partir da curva ROC, quando a fratura apresenta uma angulação em valgo $\geq 17^{\circ}$, é mais provável que seja uma fratura tipo IV. A angulação de flexão do fragmento distal, embora não 
estatisticamente significativa $(p<0,05)$, teve uma especificidade de $100 \%$. Uma fratura que apresenta uma angulação de flexão de $10^{\circ}$ é mais provável que seja do tipo IV. Mitchell et al., em seu estudo, determinaram vários parâmetros radiológicos pré-operatórios na previsão de fraturas tipo IV. ${ }^{5}$ Observou-se no estudo uma sobreposição significativa entre as fraturas tipo IV e o tipo de flexão das fraturas supracondilares. Também se observou angulação em valgo, translação lateral e aposição óssea como outros fatores na previsão de fraturas tipo IV. O presente estudo encontrou apenas a angulação em valgo como estatisticamente significativa. Outros fatores, como translação lateral e aposição óssea, não foram estatisticamente significativos.

As limitações do presente estudo são sua natureza retrospectiva e seu tamanho amostral. Outra limitação inerente do presente estudo é a falta de um teste diagnóstico padrão-ouro no diagnóstico de fraturas tipo IV. A análise de regressão multivariada não pôde ser realizada devido ao tamanho da amostra. Outra limitação importante do presente estudo é que nem todos os casos foram operados pelo cirurgião ortopédico pediátrico, mas por colegas ortopédicos pediátricos. Esse fator pode ter influência na classificação da fratura. A força do presente estudo é que ele é um dos poucos estudos determinando fatores preditivos para fraturas tipo IV. O presente estudo também é o primeiro a empregar curvas ROC para determinar um valor de corte para fraturas tipo III e IV. Os parâmetros radiográficos foram determinados por dois observadores experientes (Singh G. e Singh V.), aumentando a possibilidade de generalização em comparação com uma única abordagem observadora.

\section{Conclusão}

Concluindo, o presente estudo encontrou vários fatores préoperatórios associados às fraturas do tipo IV de Gartland. Fatores não radiográficos, como o aumento da duração da lesão hospitalar, tentativas prévias de redução fechada, e parâmetros radiográficos como valgo e angulação de flexão foram mais propensos a estarem associados a fraturas tipo IV.

Conflito de Interesses

Os autores não têm conflito de interesses a declarar.

\section{Referências}

1 Vaquero-Picado A, González-Morán G, Moraleda L. Management of supracondylar fractures of the humerus in children. EFORT Open Rev 2018;3(10):526-540

2 Cheng JC, Lam TP, Maffulli N. Epidemiological features of supracondylar fractures of the humerus in Chinese children. J Pediatr Orthop B 2001;10(01):63-67

3 Barton KL, Kaminsky CK, Green DW, Shean CJ, Kautz SM, Skaggs DL. Reliability of a modified Gartland classification of supracondylar humerus fractures. J Pediatr Orthop 2001;21(01):27-30

4 Leitch KK, Kay RM, Femino JD, Tolo VT, Storer SK, Skaggs DL. Treatment of multidirectionally unstable supracondylar humeral fractures in children. A modified Gartland type-IV fracture. J Bone Joint Surg Am 2006;88(05):980-985

5 Mitchell SL, Sullivan BT, Ho CA, Abzug JM, Raad M, Sponseller PD. Pediatric Gartland Type-IV Supracondylar Humeral Fractures Have Substantial Overlap with Flexion-Type Fractures. J Bone Joint Surg Am 2019;101(15):1351-1356

6 Silva M, Cooper SD, Cha A. The Outcome of Surgical Treatment of Multidirectionally Unstable (Type IV) Pediatric Supracondylar Humerus Fractures. J Pediatr Orthop 2015;35(06):600-605 